\title{
Crystallization Kinetics and Polymorphic Transformations in Polybutene-1
}

\author{
J. Powers, J. D. Hoffman, J. J. Weeks, and F. A. Quinn, Jr.
}

(April 1, 1965)

\begin{abstract}
When subcooled from the melt to any temperature between about 90 to $110^{\circ} \mathrm{C}$, polybutene-1 transforms at a readily measurable rate to a crystalline phase denoted "form 2," which is typically spherulitic. The kinetics of this process have been measured by optical and dilatometric techniques. The results are consistent with the concepts that the initiation is heterogeneous, that the initiation is followed by spherical growth (Avrami $n=3$ ), and that the growth rate is nucleation controlled. Values are obtained for the surface free energies and related quantities that are consistent with crystallization by chain folding: $\sigma=7.2 \mathrm{erg} /$ $\mathrm{cm}^{2}$ or $7.2 \mathrm{~mJ} / \mathrm{m}^{2}, \sigma_{e}=15.5 \mathrm{erg} / \mathrm{cm}^{2}$ or $15.5 \mathrm{~mJ} / \mathrm{m}^{2}$, and work of chain folding $q=1.7 \times 10^{-13}$ $\mathrm{erg} /$ fold. (For comparison, $q$ is $2.1 \times 10^{-13} \mathrm{erg} /$ fold for polyethylene.) The work of chain folding of polybutene-1 is also compared with that of polychlorotrifluoroethylene, and it is shown that an increase of $q$ is connected with an increase of equilibrium melting temperature. Crystal form 2 subsequently converts slowly near room temperature to a crystalline phase denoted "form 1" of different helicity and density, and the rate of this crystal-crystal transformation is also studied. The Avrami parameter for the form $2 \rightarrow$ form 1 process is $n=2$. The process appears to be nucleation controlled. It is shown that form 1 is, everywhere below its melting point, the stable form. The equilibrium melting temperature for form 2 is estimated to be about $128^{\circ} \mathrm{C}$, and for form 1 about $141^{\circ} \mathrm{C}$. A degree of crystallinity scale based on specific volume is established. The degree of crystallinity of form 2 is about 52 percent, and after conversion to form 1, the crystallinity is about $7 \overline{7}$ percent.
\end{abstract}

\section{Introduction}

Many polymers exhibit two or more polymorphic crystalline forms. The existence of three crystalline forms of polytetrafluoroethylene $[1,2,3],{ }^{1}$ which under conditions approaching equilibrium are bounded by sharp phase transitions near 20 and $30^{\circ} \mathrm{C}$, is perhaps the most familiar example. In the particular case just cited, the phase transitions are first-order crystalcrystal transitions, the transition temperatures having a truly thermodynamic significance in the same sense as does the melting point. Another example of a first-order crystal-crystal transition is the familiar rotational transition that occurs in the $n$-paraffins with chains containing 22 to 44 carbon atoms.

A change of phase of quite a different class than that described above can occur in polymers and molecular crystals generally. For purposes of clarity, these are denoted "transformations" in this paper. These phase changes occur under conditions departing considerably from equilibrium, and take place over an extended temperature range. A typical example is the formation of a crystalline phase from the subcooled liquid phase over a range of temperature. In this paper, the rate of transformation of the subcooled liquid phase of polybutene- 1 to a crystalline form designated "form 2" is studied in detail in the range of 90 to $110{ }^{\circ} \mathrm{C}$. This process occurs with the formation of typical spherulites. A crystal-crystal transformation in the same general class also occurs in polybutene- 1 . In this trans-

${ }_{1}$ Figures in brackets indicate the literature references at the end of this paper. formation, form 2 crystal converts to form 1 crystal at variable but measurable rates between 10 and $60{ }^{\circ} \mathrm{C}$. This crystal-crystal transformation is studied. As noted below, this transformation involves a change in the characteristics of the helix conformation. The melting points of the two crystalline forms are investigated. Natta [4] has given a general discussion of the origin of different crystalline phases in polymeric systems.

Earlier investigations of the transformation of form 2 to form 1 have revealed a number of facts of interest. The form $2 \rightarrow$ form 1 transformation rate is accelerated by uniaxial orientation or the application of hydrostatic pressure $[5,6]$. X-ray diffraction data indicate that the polybutene-1 chain in form 5 assumes the conformation of an $11_{3}$ helix [7] and in form 1 a $3_{1}$ helix [8]. A third crystalline form [5, 9] has been found on precipitating polybutene- 1 from solution. This latter form is reported to be stable over the period of at least several months if a temperature of roughly $100{ }^{\circ} \mathrm{C}$ is not exceeded [10]. The helicity of the polybutene- 1 chain in this form has not yet been reported.

Volume dilatometry was used to study both the isothermal rate of formation of form 2 from the subcooled liquid, and the kinetics of the ensuing transformation of form 2 to form 1. Approximate melting points of the form 2 and form 1 polymorphs were also determined dilatometrically. Dilatometric data were complemented by nucleation and growthrate measurements carried out on a hot stage microscope. Microscopical data help to elucidate the type of nucleation mechanism, the morphology of polycrystalline entities, and the rate of growth of polycrystalline aggregates as the subcooled polymer 
liquid transforms to the crystalline form 2 at a given temperature below the polymer melting point.

\section{Experimental Procedure}

\subsection{Sample}

The material used in this study was furnished by the Phillips Petroleum Company and was labeled Butene-1 (11272-38-1). Part of the material was used as received. A second portion was placed in a soxhlet extractor and left in contact with boiling ether $\left(T_{b}=35{ }^{\circ} \mathrm{C}\right)$ for 28 days. The initial weight of the latter material was $6.00 \mathrm{~g}$; the weight of the residue after extraction was $5.89 \mathrm{~g}$ and that of the extracted material $0.11 \mathrm{~g}$.

The intrinsic viscosities of the two samples were measured in $n$-nonane at $80^{\circ} \mathrm{C}$. Molecular weights were calculated from intrinsic viscosity data using the Krigbaum-Kurz-Smith relation [11]. (Professor Krigbaum has informed us of an error in the equations as published. The negative exponent in eqs (10) and (11) of reference [11] should read 5 rather than 3 . The corrected form of eq (11) is $[\eta]=5.85 \times 10^{-5}$ $M_{w}^{0.80}$.) Intrinsic viscosity data and results are given in table 1 .

\begin{tabular}{c|r|r} 
TABLE 1. Intrinsic viscosity data and molecular we \\
\hline \hline Polybutene-1 & {$[\eta], d l / g$} & \multicolumn{1}{|c}{$M_{w}$} \\
\hline As received & 1.785 & 399,000 \\
Ether-extracted & 1.849 & 412,000
\end{tabular}

\subsection{Dilatometry}

Small amounts of sample (approximately $0.5 \mathrm{~g}$ ) were vacuum molded and then sealed in glass dilatometers with mercury as the confining liquid. The dilatometers were similar to those described by Bekkedahl [12] although constructed on a smaller scale to achieve more rapid thermal equilibrium. Tru-bore tubing ( $1 \mathrm{~mm}$ i.d.) was used in constructing the dilatometers and a correction was applied for variation in bore diameter.

Three types of dilatometer runs were made. In the first type, the samples were crystallized under specified conditions to obtain either form 1 or form 2 , and the material warmed quite slowly. Here the objective was to obtain reasonably reliable melting points for the two forms. In the second type of dilatometer run, the objective was to obtain specific volume versus temperature data on forms 1 and 2 under conditions such that the degree of crystallinity did not change appreciably with temperature. This was accomplished by making the crystal form required, and measuring the specific volume curve using fairly rapid warming. In the third type of run, crystallization was carried out isothermally, and the decrease of specific volume studied as a function of time. In this case the objective was to establish the shape of the isotherms and the kinetics of the bulk crystallization process.
The melting point of form 1 for a specimen crystallized near room temperature for a period of 1 week to several months lies in the range $T_{m}=128^{\circ}$ to $T_{m}=132{ }^{\circ} \mathrm{C}$ when the melting is carried out at a moderate rate. A run of this general type is shown in figure 1. A higher melting point for form 1 is easily attained. If in the warming run the specimen is held overnight just below the melting temperature, a melting point as high as $T_{m}=137^{\circ} \mathrm{C}$ is found.

Form 2 is produced when the polymer is crystallized from the melt in the vicinity of 90 to $100{ }^{\circ} \mathrm{C}$. For a specimen crystallized 4,000 min at $100{ }^{\circ} \mathrm{C}$, fairly rapid melting gave $T_{m} 120{ }^{\circ} \mathrm{C}$. When the specimen crystallized at a higher temperature $\left(112{ }^{\circ} \mathrm{C}\right)$, a melting point of $122^{\circ} \mathrm{C}$ was found.

It has been established that the equilibrium melting temperature of a polymer, $T_{m}^{0}$, is somewhat above the highest melting point actually observed even in a very slow melting run. This occurs because the lamellar crystals are thin. A full discussion has been given in a recent review [13]. It has been found that $T_{m}^{0}$ is usually roughly $6{ }^{\circ} \mathrm{C}$ above the melting point found in a moderately slow warming run of the type used here. ${ }^{2}$ Accordingly, we would estimate that $T_{m}^{0}$ is $128 \pm 2{ }^{\circ} \mathrm{C}$ for form 2 . By similar reasoning, it is estimated that $T_{m}^{0}$ is $141 \pm 2{ }^{\circ} \mathrm{C}$ for form 1.

The specific volume data used for the degree of crystallinity calculations are shown in figure 1 . Form 2 was produced by subcooling the melt to $100{ }^{\circ} \mathrm{C}$, and allowing the sample to remain at that temperature for 3 days. The sample was then quenched to $25^{\circ} \mathrm{C}$, and the specific volume data obtained with fairly rapid heating. Prolonged storage below $40{ }^{\circ} \mathrm{C}$ was avoided in order to prevent a significant conversion of form 2 to form 1 . Similarly, prolonged storage at higher temperatures had to be avoided to prevent additional conversion of subcooled liquid to form 2. Form 1 was produced by subcooling a specimen from the melt to room temperature and storing for 1 week. This allowed the sequences liquid $\rightarrow$ form 2 and form $2 \rightarrow$ form 1 to take place. The specific volume data were then obtained with fairly rapid warming. The data are shown in figure 1.

\subsection{Hot Stage Microscopy}

A thermistor was attached to a standard Kofler hot stage, and the sample temperature was monitored by measuring the resistance of the thermistor with a Wheatstone bridge circuit. The thermistor was calibrated by a previous direct measurement of the temperature inside a duplicate sample using a copper-constantan thermocouple and a precision potentiometer. The temperature was known to within $\pm 0.2{ }^{\circ} \mathrm{C}$.

The samples were first melted by heating to between 160 and $180{ }^{\circ} \mathrm{C}$ on a Kofler hot bench and then rapidly transferred to the surface of the hot

If very slow warming is used, the observed melting point approaches to within about $4^{\circ} \mathrm{C}$ of $T_{m}^{\circ}$ 


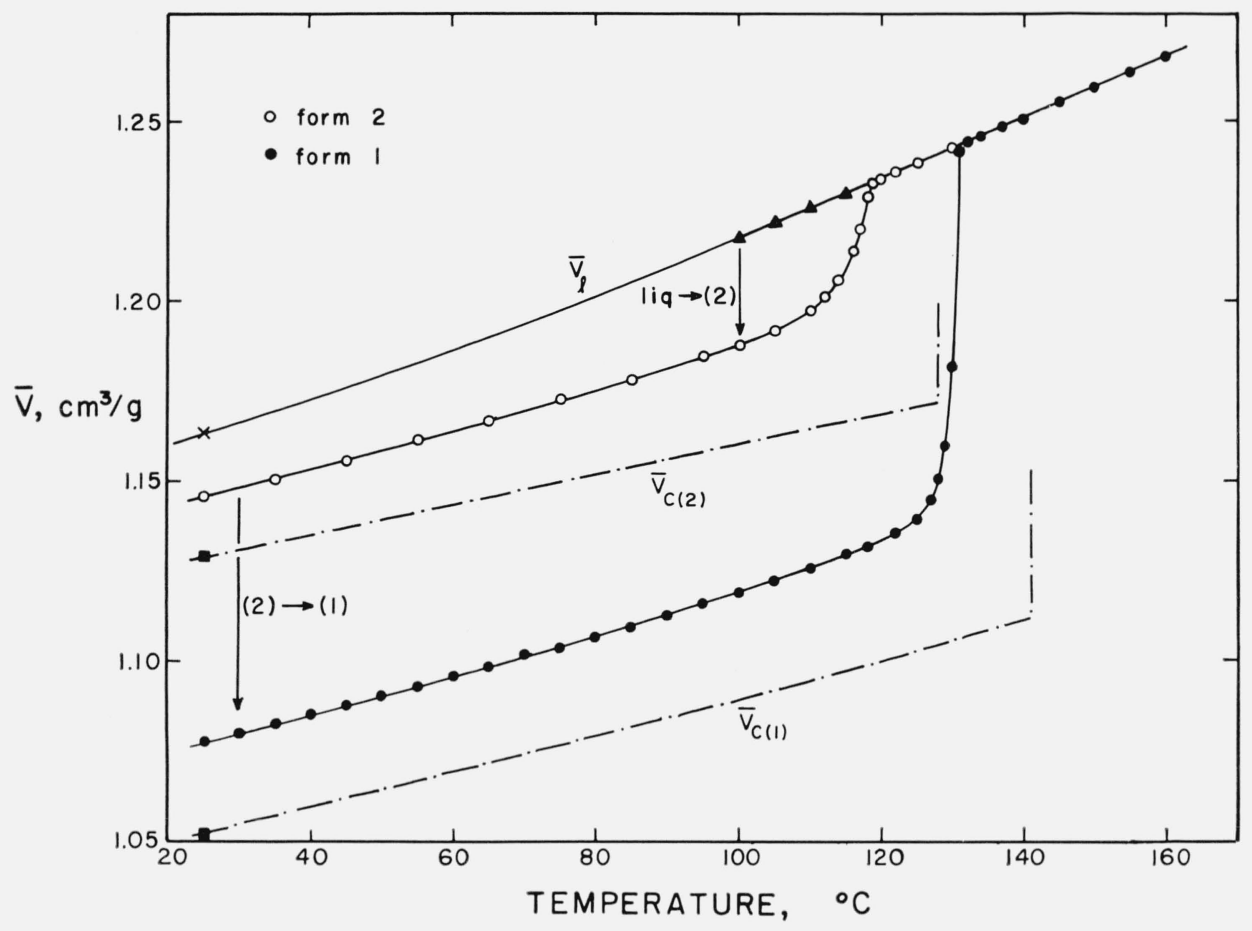

Figure 1. Specific volume-temperature curves for two polybutene-1 polymorphs ("as received" material).

F orm 1 crystallized at $25{ }^{\circ} \mathrm{C}$, form 2 at $100{ }^{\circ} \mathrm{C}$. Data obtained under conditions

of fairly rapid warming.

X-ray specific volume of pure crystal of form 1 and form 2 at $25^{\circ} \mathrm{C}$.

Derived value of subcooled liquidus at $25^{\circ} \mathrm{C}$.

-. - Estimated value of pure crystal specific volume for forms 1 and $2, \bar{V}_{c(1)}$ and $\left.\bar{V}_{c(2)}\right)$

stage. The process of crystallization was characterized by photomicrographs taken at time intervals appropriate for the particular temperature of the transformation.

The diameter of each spherulite was measured along two orthogonal directions with a comparator whose precision was $1 \mu$. Magnification was computed from measurements of a micrometer slide whose smallest division was $0.01 \mathrm{~mm}$. The slide was photographed with the same objective-ocular combination as was used for the spherulites.

\section{Results}

\subsection{Dilatometric Observations of Rate Effects}

The isothermal crystallization time plots (fig. 2) at various temperatures show two ranges of different behavior. The upper temperature range (90 to $110{ }^{\circ} \mathrm{C}$ ) displays a marked rate-dependence on temperature, while the lower range $\left(10\right.$ to $\left.60{ }^{\circ} \mathrm{C}\right)$ displays considerably less dependence. Comparison of crystallization half-time data (table 2) affords a quantitative means of characterizing the two regions. The half times for the "as received" polybutene-1 samples range from $3.5 \mathrm{~min}$ at $90{ }^{\circ} \mathrm{C}$ to $2200 \mathrm{~min}$ at $110{ }^{\circ} \mathrm{C}$ for the upper range, while for the lower

TABLE 2. Comparison of isothermal crystallization rate halftimes ( $\left.\mathrm{t}_{1 / 2}\right)$ for "as received" and for ether-extracted polybutene-1 as a function of temperature

\begin{tabular}{c|c|c}
\hline \hline \multirow{3}{*}{ Temperature } & \multicolumn{2}{|c}{$t_{1 / 2}$ minutes } \\
\cline { 2 - 3 } & As received & Ether-extracted \\
\hline & & \\
\hline & & \\
& Phase transformation & \\
110 & liquid $\rightarrow$ form 2 & \\
105 & 2200 & 1400 \\
100 & 170 & 100 \\
95 & 26 & 18 \\
90 & 8 & 6 \\
& 3.5 & 3 \\
\hline
\end{tabular}

Form $2 \rightarrow$ form 1

\begin{tabular}{l|l|c}
\hline & 3900 & 3750 \\
40 & 2400 & 2600 \\
35 & 1750 & 1600 \\
30 & 1400 & $-\ldots-$ \\
10 & 1475 & \\
\hline
\end{tabular}




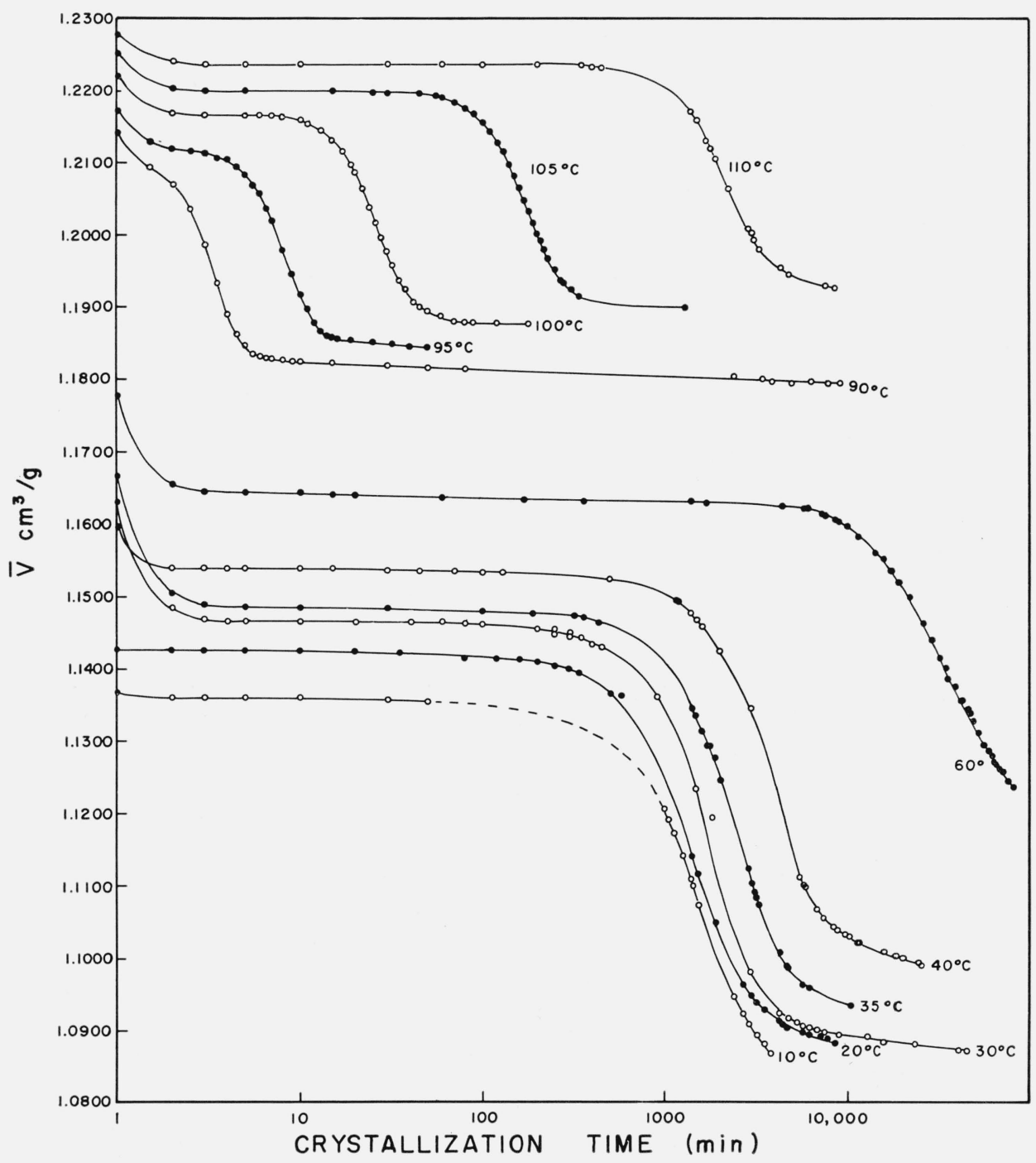

FIGURE 2. Isothermal specific volume-time plots for "as received" polybutene-1.

(a) Upper curves $\left(90-110^{\circ} \mathrm{C}\right)$ supercooled liquid $\rightarrow$ form 2 .

(b) Lower curves $\left(10-60^{\circ} \mathrm{C}\right)$ form $2 \rightarrow$ form 1 .

region the half-times are $1400 \mathrm{~min}$ at $20{ }^{\circ} \mathrm{C}$ to 3900 min at $40^{\circ} \mathrm{C}$. The corresponding half times for the ether-extracted sample are $3 \mathrm{~min}$ at $90{ }^{\circ} \mathrm{C}$ to 1400 min at $110{ }^{\circ} \mathrm{C}$ for the upper region, and for the lower region, $1600 \mathrm{~min}$ at $20^{\circ} \mathrm{C}$ and $3750 \mathrm{~min}$ at $40{ }^{\circ} \mathrm{C}$.

It has already been noted that polybutene-1 exhibits two melting points (fig. 1). Which melting point is found depends on the temperature range in which the sample is crystallized. If crystallized in the upper range $\left(90\right.$ to $110{ }^{\circ} \mathrm{C}$ ), the material melts between 118 and $122{ }^{\circ} \mathrm{C}$, and if crystallized and stored in the lower range $\left(10\right.$ to $\left.40^{\circ} \mathrm{C}\right)$, the sample melts between 128 and $132{ }^{\circ} \mathrm{C}$ for relatively slow heating rates. ${ }^{3}$ Thus, in line with Boor and Mitchell's [14] report of similar melting points, the lower melting form is identified as Natta's form 2 and the higher melting form as his form 1 . Since the specific volumes (fig. 2) on the induction portions of the isothermal crystallization curves have the same values as those of the liquidus line (fig. 1) in the range 90 to $110{ }^{\circ} \mathrm{C}$, the transformation in this range therefore represents a subcooled liquid $\rightarrow$ crystal (form 2) change. For the lower region $\left(10\right.$ to $\left.60{ }^{\circ} \mathrm{C}\right)$ the specific volumes on the induction part of the crystallization curve have values lower than those of the liquidus curve in the corresponding

3 For a given crystal form, a higher melting point is obtained the higher the transformation temperature. 
region (fig. 1). ${ }^{4} \quad$ The lower region change represents a crystal $\rightarrow$ crystal transformation with form 2 changing into the more stable form 1 . This point will be discussed further in section 4.3.

\subsection{Degree of Crystallinity}

The degree of crystallinity of form 1 was calculated from

$$
\chi_{1}=\frac{\bar{V}_{l}-\bar{V}_{s}}{\bar{V}_{l}-\bar{V}_{c(1)}}
$$

where $\bar{V}_{l}$ represents the specific volume of the liquid phase, $\bar{V}_{s}$ the specific volume of the sample, and $\bar{V}_{c(1)}$ the specific volume of the pure form 1 crystal phase (see fig. 1). In a similar manner, the degree of crystallinity of form 2 was calculated from

$$
\chi_{2}=\frac{\bar{V}_{l}-\bar{V}_{s}}{\bar{V}_{l}-\bar{V}_{c(2)}}
$$

where $\bar{V}_{c(2)}$ represents the specific volume of the pure form (2) crystal. As has been noted many times, for example in [15], these expressions depend on the assumption that the volumes of the liquid and crystalline phases are additive.

In the present case the known quantities in eqs (1) and (2) are $\bar{V}_{s}$ at any desired temperature, $\bar{V}_{c(1)}$ and $\bar{V}_{c(2)}$ at $25{ }^{\circ} \mathrm{C}$ from x-ray data, and $\bar{V}_{l}$ at 100 ${ }^{\circ} \mathrm{C}$. The situation is then that at $25{ }^{\circ} \mathrm{C}, \bar{V}_{s}$ and $\bar{V}_{c(1)}$ or $\bar{V}_{c(2)}$ are known, but $\bar{V}_{l}$ is not, while at 100 ${ }^{\circ} \mathrm{C}, \bar{V}_{s}$ and $\bar{V}_{l}$ are known, but $\bar{V}_{c(1)}$ or $\bar{V}_{c(2)}$ are not. The value of $\bar{V}_{l}$ at $25{ }^{\circ} \mathrm{C}$ might be obtained by downward extrapolation of the liquidus data, but this procedure is rather inaccurate because the supercooled liquidus often shows curvature. Accordingly, we proceeded by developing a simple method to obtain $\bar{V}_{c(2)}$ at $100{ }^{\circ} \mathrm{C}$. Given this, $\chi_{2}$ and $\bar{V}_{l}^{25}$ can readily be calculated using eq $(2)$. Then $\chi_{1}$ can be obtained using eq (1) with the value of $\bar{V}_{l}^{25}$ obtained from the form 1 calculation, and the known values of $\bar{V}_{c(1)}^{25}$ and $\bar{V}_{s}$ for form 1 .

The method for obtaining $\bar{V}_{c(2)}^{100}$ for form 2 is now given. On the basis of the additivity of the volumes of the liquid and crystalline phases [15], it is readily shown that

$$
\left(\bar{V}_{l}^{100}-\bar{V}_{s}^{100}\right) \Delta T \alpha_{l}^{100}-\left(\bar{V}_{l}^{100}-\bar{V}_{s}^{100}\right)\left(\bar{V}_{c}^{100}-\bar{V}_{c}^{25}\right)
$$

$$
=\Delta T\left(\alpha_{l}^{100}-\alpha_{s}^{100}\right) \bar{V}_{l}^{100}-\Delta T\left(\alpha_{l}^{100}-\alpha_{s}^{100}\right) \bar{V}_{c}^{100}
$$

where $\bar{V}_{c}^{100}$ refers to $\bar{V}_{c(2)}^{100}$ and $\alpha=d \bar{V} / d T$. It is assumed that there is no curvature in $\bar{V}_{c(2)}$ between 25 and $100{ }^{\circ} \mathrm{C}$. The quantity $\Delta T$ is $75^{\circ} \mathrm{C}$ in the present case, but other temperature intervals could be chosen. In eq (3), the quantities $\alpha_{l}^{100}$ and $\alpha_{s}^{100}$

4 The method by which the supercooled liquidus curve was established at low temperature is discussed subsequently. are known, as are all the other quantities except $\bar{V}_{c(2)}^{100}$. Given $\bar{V}_{c(2)}^{100}$ as calculated by eq (3), it is a simple matter to calculate $\chi_{2}$ at $100^{\circ} \mathrm{C}$ using eq (2). Then assuming $\chi_{2}$ is constant with temperature, $\bar{V}_{l}$ can be calculated at $25^{\circ} \mathrm{C}$ using eq (2).

The above method should only be applied where the $\bar{V}_{s}$ data are nearly linear in the temperature range chosen if the sample is thought to be highly crystalline. This condition is not met by the data on form 1. The data on form 1 imply more curvature in $\bar{V}_{c(1)}$ than is allowable for this method to be accurately applicable.

\begin{tabular}{|c|c|c|c|c|c|c|}
\hline \multirow{2}{*}{ Polymorph } & \multirow{2}{*}{ Crystal unit cell } & \multicolumn{3}{|c|}{ Unit cell parameters, } & \multirow{2}{*}{$\begin{array}{l}\text { Crystal } \\
\text { density }\end{array}$} & \multirow{2}{*}{$\begin{array}{l}\text { Chain } \\
\text { confor- } \\
\text { mation }\end{array}$} \\
\hline & & $a$ & $b$ & $c$ & & \\
\hline $\begin{array}{l}\text { Form 1 } \\
\text { Form 2 }\end{array}$ & $\begin{array}{l}\text { Rhombohedral }[17,18] \\
\text { Tetragonal a }[19] \\
\text { Tetragonal a }[7]\end{array}$ & \begin{tabular}{|l}
17.7 \\
b 14.89 \\
14.85
\end{tabular} & $\begin{array}{l}17.7 \\
14.89 \\
14.85\end{array}$ & $\begin{array}{l}6.50 \\
20.87 \\
20.6\end{array}$ & $\begin{array}{c}g / \mathrm{cm}^{3} \\
0.9507 \\
0.0 .886 \\
0.90\end{array}$ & $\begin{array}{r}3{ }_{1} \\
11_{3} \\
11_{3}\end{array}$ \\
\hline
\end{tabular}

The $x$-ray data used to estimate the densities of pure form 1 and form 2 are collected in table 3 .

TABLE 3. Crystallographic data for polybutene-1 polymorphs

a Unit cell contains four polymer chains.

b The thickness of a monomolecular layer is $7.445 \AA$ on the (100) growth plane. - Recalculated.

The results of the degree of crystallinity calculations are shown in table 4 . The probable error in the degree of crystallinity is estimated to be about 4 percent.

The liquidus value obtained at $25{ }^{\circ} \mathrm{C}$ is slightly above (about $0.009 \mathrm{~cm}^{3} / \mathrm{g}$ ) that obtained by a linear extrapolation of the actual liquidus data in the 100 to $160{ }^{\circ} \mathrm{C}$ range. This is normal behavior. The $d \bar{V} / d T$ values obtained for the pure crystal for forms 1 and 2 are in the usual range for high polymers. Note that $\alpha_{c(1)}$ depends on temperature.

The results listed in table 4 may be interpreted as follows. The subcooled liquid is converted to form 2 to a maximum observable extent of about 52 percent. The conversion is slow at high temperatures, but below $90{ }^{\circ} \mathrm{C}$ the transformation becomes so fast that the maximum conversion rate cannot be measured. Hence form 2 appears straightaway to practically its full extent in a sample quenched from the melt to room temperature. Form 2 then proceeds to slowly convert to form 1 at a rate which is a maximum near room temperature. After the form $2 \rightarrow$ form 1 transformation has essentially stopped, the mass fraction that is crystalline is about 77 percent. This together with other evidence implies that practically all of form 2 converts into form 1 , and further implies that some small amount of the remanent subcooled liquid crystallizes during the form $2 \rightarrow$ form 1 transformation.

The above conclusions are consistent with infrared and $x$-ray diffraction data that initially show only absorption phenomena characteristic of form 2 after polybutene-1 is cooled from above its melting point to room temperature, and only form 1 after prolonged storage at the same temperature. 
TABLE 4. Degree of crystallinity

Form 2

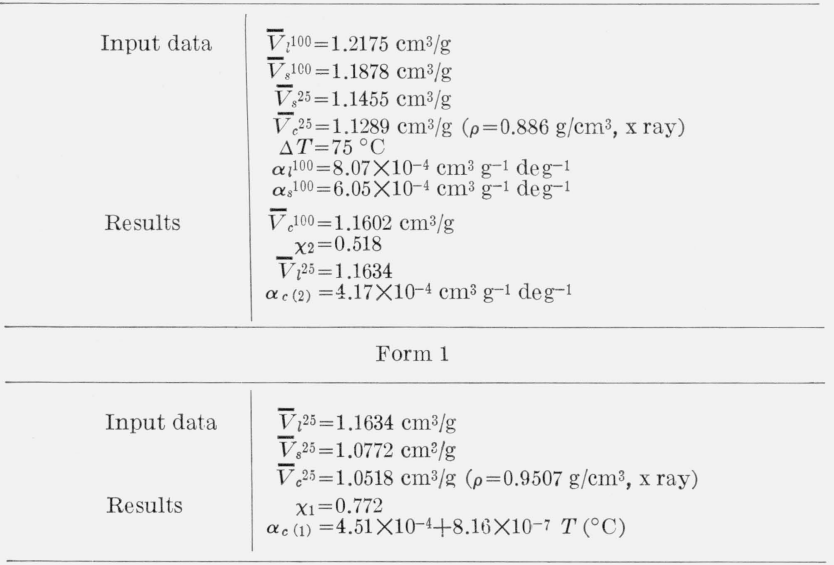

Boor and Mitchell [14] interpret their torsional damping data as indicating that but little crystallization of liquid occurs during the form $2 \rightarrow$ form 1 transformation. Our results imply that some amorphous material does in fact crystallize during the $2 \rightarrow 1$ process. Zannetti, Manaresi, and Buzzoni [5] have studied the polymorphic behavior of polybutene-1 as a function of temperature and pressure by means of $x$-ray diffraction and density techniques. Their density data are stated to imply a degree of crystallinity of between 40 and 50 percent for form 1. Wilski and Grewer [16] find using their specific volume data that form 1 exhibits a degree of crystallinity of $\chi_{1}=0.76$, which is very close to that found in the present study.

Wilski and Grewer's calorimetric data [16] may be interpreted to mean that $\chi_{2}$ is close to 0.49 (see footnote in table 7), which is in good agreement with our value of 0.52 . Their specific volume data for form 2, when recomputed using our $\bar{V}_{c(2)}$ and $\bar{V}_{l}$ values, indicate that $\chi_{2}$ is 0.47 . Wilski and Grewer assumed in their paper that the degree of crystallinity of form 2 was the same as that of form 1, i.e., 0.76. Our interpretation of their calorimetric data, and our dilatometric data, indicates that $\chi_{2}$ is actually nearer to 0.5 .

By crystallizing polybutene-1 under 800 bars pressure, we have succeeded in attaining a degree of crystallinity of over 80 percent by the dilatometric method (form 1). This is comparable to the degree of crystallinity obtained by the dilatometric method for well annealed samples of polyethylene, which commonly yield $\chi$ values near 0.80 to 0.85 (see for example reference [13]). In the case of polybutene, the true degree of crystallinity is probably considerably higher than 0.8 , because voids artificially lower $\chi$. For example, a careful analysis of heat capacity data, which is insensitive to the effect of voids, yields a $\chi$ value in excess of 0.95 for a well-annealed polyethylene specimen identical to that which gives $\chi=0.80$ by the dilatometric method [13]. The implication is that the polybutene-1 studied here may be regarded as a highly crystalline polymer that does not exhibit any serious degree of atacticity.

\subsection{Avrami Parameter}

The Avrami equation is commonly used to fit and to interpret isothermal crystallization-time plots and may be represented as [20]

$$
\frac{h_{t}-h_{\infty}}{h_{0}-h_{\infty}}=\exp (-K t)^{n},
$$

where $h_{t}$ represents the mercury height at time $t, h_{0}$ the height during the induction period, $h_{\infty}$ the height after the isotherm has leveled off, and $K$ is a parameter which includes growth rate, nucleation rate and temperature terms. The parameter $n$ is a dimensionless number ranging between 1 and 4 . The value of $n$ can under appropriate circumstances be interpreted as signifying the type of initiation and the dimensionality of the developing crystalline phase.

The parameter $n$ was calculated from the differentiated form of eq (5)

$$
n=\frac{t\left(d h_{t} / d t\right)}{\left(h_{\infty}-h_{t}\right)\left\{\ln \left[\frac{h_{0}-h_{\infty}}{h_{t}-h_{\infty}}\right]\right\}} .
$$

The average magnitude of $n$ was not appreciably affected by the choice of $h_{\infty}$. Results are shown in table 5. The estimated uncertainties noted in table 5 are average deviations from the mean values of $n$.

\subsection{Seeding Experiment}

Large numbers of form 1 nuclei were prepared by heating a form 1 specimen to a temperature near the melting point so that the specific volume was just below the liquidus. Then the specimen was cooled to $100{ }^{\circ} \mathrm{C}$ where form 2 ordinarily grows from the melt and the crystallization was allowed to proceed. The melting curve of the specimen was then obtained. It was found that the newly formed crystals melted in the same range as form 2 obtained without seeding.

TABLE 5. Avrami parameter for polybutene-1 transformations

\begin{tabular}{c|c|c}
\hline \hline Temperature & Subcooled liquid $\rightarrow$ form 2 & $n$ \\
\cline { 3 - 3 }${ }^{\circ} C$ & & \\
110 & & $2.9 \pm 0.3$ \\
105 & $3.2 \pm 0.2$ \\
100 & $3.3 \pm 0.4$ \\
95 & $3.8 \pm 0.4$ \\
\hline
\end{tabular}

Form $2 \rightarrow$ form 1

\begin{tabular}{l|l}
\hline 40 & \\
35 & $1.8 \pm 0.2$ \\
30 & $2.2 \pm 0.3$ \\
20 & $2.2 \pm 0.6$ \\
10 & $1.5 \pm 0.5$ \\
& $2.0 \pm 0.3$ \\
\hline
\end{tabular}



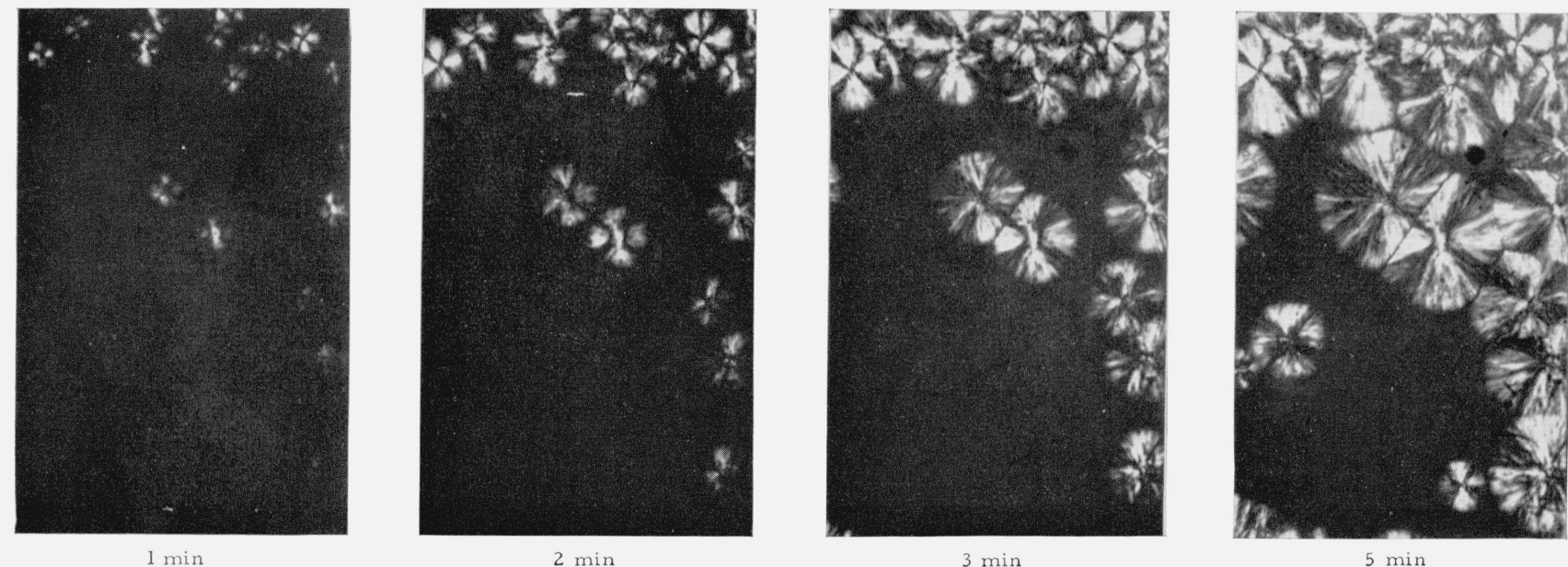

Figure 3. Photomicrographs showing growth sequence for polybutene-1 spherulites crystallized at $90.2^{\circ} \mathrm{C}$

Specimen cooled from $T_{1}=160^{\circ} \mathrm{C}$. Magn. $108 \times$. Crossed polaroids.

This result is to be expected from the results on the kinetics of crystallization: the growth rate of form 1 at $100{ }^{\circ} \mathrm{C}$ is by all accounts exceedingly slow, while form 2 nucleates itself and grows at a measurable rate at this temperature.

\subsection{Microscopical Observations}

Observations of spherulitic nucleation and growth rates were made over a temperature range of approximately 90 to $107{ }^{\circ} \mathrm{C}$. Above $107{ }^{\circ} \mathrm{C}$, crystallization occurred too slowly to be feasible in terms of time and temperature control of the equipment. Below $90{ }^{\circ} \mathrm{C}$, crystallization occurred too rapidly to be followed. Thus, the development of spherulites could not be studied at low temperatures where the growth rate is diffusion controlled. Some isolated observations were made on spherulitic films formed at $105{ }^{\circ} \mathrm{C}$ and kept at room temperature for several months. These were compared with spherulites freshly prepared at the same temperature. No differences in optical appearance as regards texture or character of the birefringence could be discerned between the two sets of spherulites.

All spherulites observed displayed a coarse fibrillar texture with some tendency for random mixing of positively and negatively birefringent areas (figs. 3 and 4). Their appearance is thus similar to the mixed form polypropylene spherulites reported by Padden and Keith [21]. Spherulites nucleated and grown at $90{ }^{\circ} \mathrm{C}$ (fig. 3) displayed a more typical structure (i.e., the maltese cross is easily distinguished) than did those formed at $107^{\circ} \mathrm{C}$ (fig. 4). No ringed or other types of spherulites with well developed optical patterns, however, were observed in this study. Since almost all spherulites first appeared over a narrow time interval, we conclude that the dominant mode of initiation is heterogeneous in origin. Some growth sequences (center left fig. 3, compare $5 \mathrm{~min}$ with $3 \mathrm{~min}$ photomicrograph) showed a few spherulites appearing at later times.

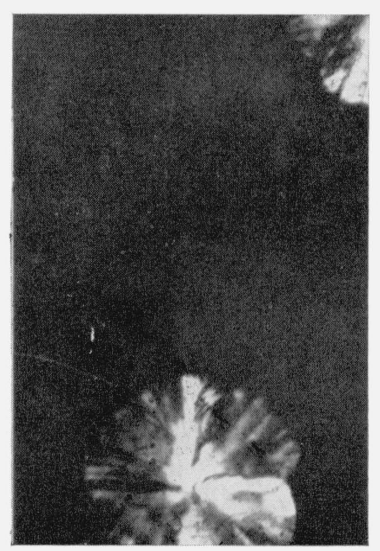

$18 \mathrm{hr} 10 \mathrm{~min}$

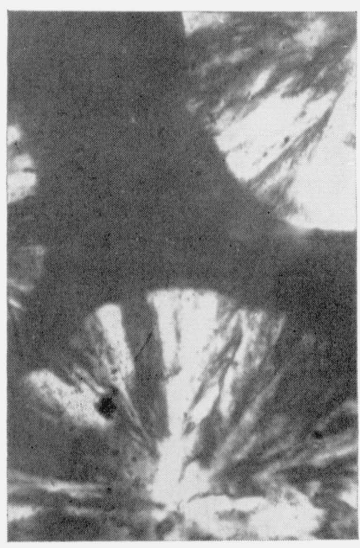

$24 \mathrm{hr}$
Figure 4. Photomicrographs showing growth sequence for polybutene-1 spherultes crystallized at $106.7^{\circ} \mathrm{C}$.

Specimen cooled from $T_{1}=160{ }^{\circ} \mathrm{C}$. Magn. 108×. Crossed polaroids.

These spherulites are almost certainly pseudohomogeneous as defined by Hoffman and Lauritzen [22].

Spherulite radius-time plots were prepared from the photomicrographs, and radial growth rates were calculated as $G=d r / d t$, where $r$ is the radius. The results are shown in table 6. Except when the spherulites impinged, the radius-time plots were linear, showing that polybutene-1 exhibits typical lineal growth. Each $G$ value is an average for several spherulites. The radius-time line generally intersected the time coordinate at a positive value, indicating the existence of a short induction time, $\tau_{i}$. This is typical of spherulitic growth in many polymers.

The growth rate data in table 6 indicate that the spherulitic grow th process has a negative temperature coefficient, i.e., the growth is slower the higher the growth temperature. This type of temperature 
TABLE 6. Polybutene-1 isothermal spherulite growth rate ${ }^{a}$ (Supercooled liquid $\rightarrow$ form 2)

\begin{tabular}{c|c}
\hline \hline $\begin{array}{c}\text { Growth } \\
\text { temperature }\end{array}$ & $\begin{array}{c}\text { Radial growth rate, } \\
G=d r / d t\end{array}$ \\
\cline { 1 - 2 } & $\begin{array}{c}c m / s e c \\
C\end{array}$ \\
90.0 & $3.38 \times 10^{-5}$ \\
90.2 & $3.02 \times 10^{-5}$ \\
90.2 & $2.88 \times 10^{-5}$ \\
96.0 & $1.98 \times 10^{-5}$ \\
96.0 & $1.39 \times 10^{-5}$ \\
100.0 & $0.604 \times 10^{-5}$ \\
101.6 & $0.357 \times 10^{-5}$ \\
101.6 & $0.328 \times 10^{-5}$ \\
105.0 & $0.196 \times 10^{-5}$ \\
106.7 & $0.106 \times 10^{-5}$ \\
& \\
\hline
\end{tabular}

a The specimens were first heated to $T_{1}=160^{\circ} \mathrm{C}$ and then cooled to the growth temperature.

dependence implies that the grow th step is nucleation rather than diffusion controlled.

\section{Discussion}

\subsection{Subcooled Liquid $\rightarrow$ Form 2 Transformation}

The liquid $\rightarrow$ form 2 process can readily be understood in terms of current concepts of spherulitic growth with chain folding. These concepts have been discussed in a recent review [13]. Surface free energies may be calculated from spherulitic growth data for the liquid $\rightarrow$ form 2 process by use of the eqation $[13,22,23]^{5}$

$G=G_{0} \quad \exp \quad\left(-\Delta H^{*} / R T\right) \quad \exp \left[-K_{g} / T^{2}(\Delta T)\right]$

where $K_{g}$ is $4 b_{0} \sigma \sigma_{e}\left(T_{m}^{0}\right)^{2} /\left(\Delta h_{f}\right) k$ and $\Delta H^{*}$ is the activation energy of the in terfacial transport process. In the following calculation, $\Delta H^{*}$ is assigned an arbitrary value of $20 \mathrm{kcal}$, which is a sufficient approximation in the region where the growth rate has a strongly negative temperature coefficient. If the grow th rate data had extended to the maximum or to lower temperatures, for which growth rates show a positive temperature coefficient, then the $\Delta H^{*}$ term would be better approximated by a WLF type expression (see Hoffman and Weeks [23]). In the expression for $K_{g}, b_{0}$ is the thickness of a coherent surface nucleus, $\sigma$ the lateral surface free energy, $\sigma_{e}$ the end surface free energy characteristic of the chain folds, $T_{m}^{0}$ the equilibrium melting temperature, $\Delta h_{f}$ the heat of fusion and $k$ Boltzmann's constant. When $\log G+\Delta H^{*} / 2.3 R T$ is plotted against $1 / T^{2} \Delta T$ and a straight line is obtained, then grow th is governed by coherent two-dimensional growth nuclei. The slope of the line is $K_{g} / 2.303$. This type of plot is shown in figure 5 for the case $T_{m}^{0}=403^{\circ} \mathrm{K}$. The value of $K_{q}$ obtained from this plot and the corresponding value of $\sigma \sigma_{e}$ is given in table 7 .

The value of $K_{g}$ can also be estimated from the half-times for bulk crystallization given in table 2 . In this case one plots $-\log t_{1 / 2}-(1 / 3) \log \nu_{0}+\Delta H^{*} / 2.3$

${ }^{5}$ Sometimes the last term in eq (6) is written $\exp \left[-K_{g}^{\prime} / T(\Delta T)\right]$ with $K_{g}^{\prime}=$ $4 b_{0} \sigma \sigma_{e} T_{m}^{0} / \Delta h_{j} k$. This leads to only a slightly different value of $\sigma \sigma_{e}$ (see references $[22]$ and $[23]$ ).
$R T$ against $1 / T^{2}(\Delta T)$, the slope being $K_{g} / 2.303$. A plot of this type is shown in figure 5 for the case $T_{m}^{0}=403^{\circ} \mathrm{K}$. The half-times are corrected for the change in $\nu_{0}$, the number of growth centers per unit volume, by the term - (1/3) $\log \nu_{0}$. The data have also been corrected for the induction period $\tau_{i}$. This correction is small. The $K_{g}$ value obtained from the dilatometric half-times is identical within experimental error to that obtained from the spherulite growth rate data (see table 7 ).

The rate data have been analyzed using $T_{m}^{0}=$ $399^{\circ} \mathrm{K}, T_{m}^{0}=401^{\circ} \mathrm{K}$, and $403^{\circ} \mathrm{K}$ to show the effect of varying $T_{m}^{0}$ on the results. The true value of $T_{m}^{0}$ probably lies between 400 and $402{ }^{\circ} \mathrm{K}$.

The fit of the data to the theoretical expression shown in figure 5 is good for the dilatometric halftime data. In this case the same specimen was used in all the runs. The scatter is greater in the case of the radial growth data, probably because different specimens (from the same lot) were used in making a number of the runs. Nevertheless, the theory seems to provide a reasonable basis for analysis of the data within the estimated experimental error. The fit is almost equally good if $T_{m}^{0}$ is taken to be $401{ }^{\circ} \mathrm{K}$. The fit is poor if $T_{m}^{0}$ is taken to be $398^{\circ} \mathrm{K}$ or less.

Lateral surface free energy $(\sigma)$ was calculated from the equation [23]

$$
\sigma=0.10\left(b_{0}\right)\left(\Delta h_{f}\right) .
$$

Once values have been calculated for $\sigma$ and $\sigma \sigma_{e}, \sigma_{e}$ can be obtained by taking the ratio of $\sigma \sigma_{e}$ to $\sigma$. Results from these calculations are given in table 7 .
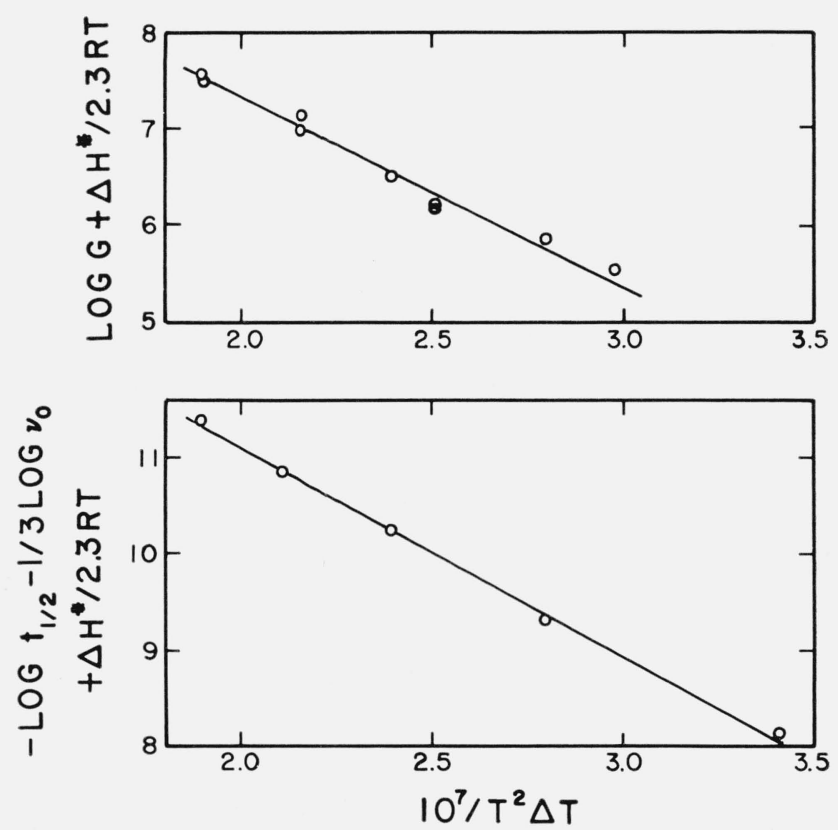

Figure 5. Determination of $\mathrm{K}_{\mathrm{g}}$ for supercooled liquid $\rightarrow$ form 2 transformation.

Upper diagram: Spherulite growth rate data. Lower diagram: Dilatometric half-time data. 
TABLE 7. Surface free energies and work of chain folding of form 2 polybutene-1

(Subcooled liquid $\rightarrow$ form 2 transformation)

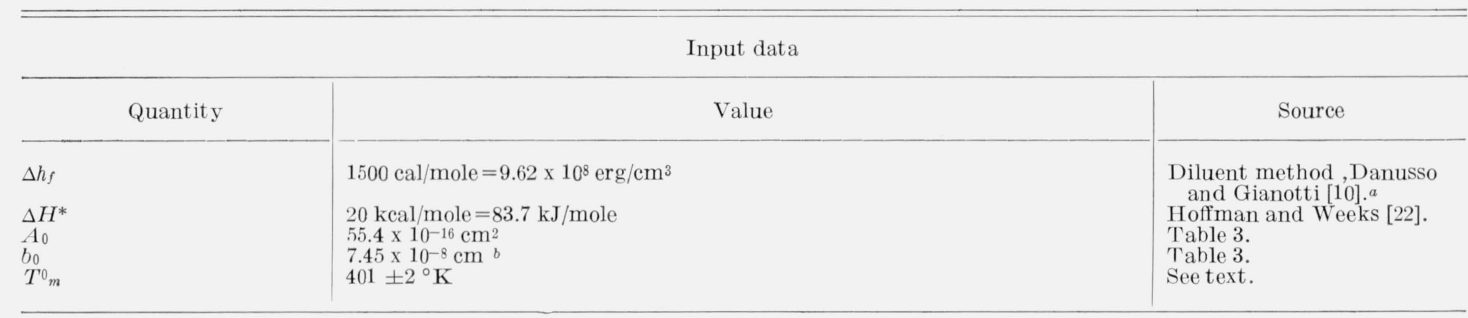

Results

\begin{tabular}{|c|c|c|c|c|}
\hline \multirow{2}{*}{ Quantity } & \multicolumn{3}{|c|}{ Value } & \multirow{2}{*}{ Source } \\
\hline & $T^{0}{ }_{m}=399^{\circ} \mathrm{K} ;\left(T_{m}+4^{\circ}\right)$ & $T^{0}{ }_{m}=401^{\circ} \mathrm{K} ;\left(T_{m}+6^{\circ}\right)$ & $T^{0}{ }_{m}=403^{\circ} \mathrm{K} ;\left(T_{m}+8^{\circ}\right)$ & \\
\hline $\begin{array}{l}K_{g}\left({ }^{\mathrm{C}} \mathrm{K}\right)^{3} \\
K_{g}\left({ }^{\mathrm{C}} \mathrm{K}\right)^{3} \\
\sigma \sigma_{e} \mathrm{erg}^{2} / \mathrm{cm}^{4} \\
\sigma \mathrm{mJ} / \mathrm{m}^{2} \text { or }\left(\mathrm{erg} / \mathrm{cm}^{2}\right) \\
\sigma_{e} \mathrm{~mJ} / \mathrm{m}^{2} \text { or }\left(\mathrm{erg} / \mathrm{cm}^{2}\right) \\
q(\mathrm{erg} / \text { fold })\end{array}$ & $\begin{array}{l}3.46 \times 10^{7} \\
3.23 \times 10^{7} \\
39.7 \\
7.16 \\
13.8 \\
1.45 \times 10^{-13}\end{array}$ & $\begin{array}{c}4.17 \times 10^{7} \\
3.79 \times 10^{7} \\
110.4 \\
7.16 \\
15.42 \\
1.71 \times 10^{-13} \\
=15.5 \pm 2.5 ; q=1.7 \pm 0.3 \times 1\end{array}$ & $\begin{array}{l}4.95 \times 10^{7} \\
4.42 \times 1 \mathrm{C}^{7} \\
128.6 \\
7.16 \\
17.96 \\
1.99 \times 10^{-13}\end{array}$ & $\begin{array}{l}\text { Dilatometric data, figure } 5 . \\
\text { Radial growth data, figure } 5 . \\
\text { From average of } K_{g} \text {. }\end{array}$ \\
\hline
\end{tabular}

$a$ Wilski and Grewer [16] give the heat of fusion as $974 \mathrm{cal} / \mathrm{mole}$ as determined calorimetrically on the assumption that their specimen was 76 percent crystalline as determined from their dilatometric crystallinity scale. According to our $\chi_{2}$ crystallinity scale, their sample was 0.47 percent crystalline, which leads to a corrected heat of fusion of $\Delta h_{f}=1570 \mathrm{cal} / \mathrm{mole}$, which is in good agreement with that obtained by Danusso and Gianotti. Wilski and Grewer give the actual heat of fusion of their form 2 specimen as $740 \mathrm{cal} / \mathrm{mole}$. Combining this with Danusso and Gianotti's value $\Delta h_{f}=1500 \mathrm{cal} / \mathrm{mole}$ gives $\chi_{2}=740 / 1500=0.493$.

$b$ Holland and Miller [26] have shown that growth occurs on the (100) plane in single crystals of form 2.

From the standpoint of the theory of chain folding, one polymer is most aptly compared to another through the work of chain folding, $q$. The value of the work of chain folding $[13,23], q$, is calculated from

$$
\sigma_{e}=q / 2 A_{0}
$$

where $A_{0}$ is the cross-sectional area of the chain. The value of $q$ for polybutene- 1 is $1.7 \times 10^{-13} \mathrm{erg} /$ fold (table 7). In table 8 , the values of $q$ and other parameters of interest are compared for polybutene-1, polyethylene [13], and polychlorotrifluoroethylene [13]. The analyses given for polyethylene and polychlorotrifluoroethylene are based on highly reliable values of $T_{m}^{0}$. The value of $q$ for polybutene- 1 is seen to be similar to that of polyethylene, but smaller than that for polychlorotrifluoroethylene. We regard the value of $q$ for polybutene- 1 to be entirely reasonable, especially in view of its general similarity to that found for polyethylene. On the basis of molecular structure, one would expect the polybutene-1 chain to have a stiffness about like that of polyethylene, but less than polychlorotrifluoroethylene. The value of $q$ for polyethylene is known on the basis of a number of fundamental considerations to represent the work of chain folding [13]. Thus, the crystallization of form 2 polybutene- 1 is consistent with a mechanism involving chain folding. At the same time, the value $\sigma_{e}=15.5$ $\mathrm{erg} / \mathrm{cm}^{2}$ for polybutene- 1 is seen to be reasonable in terms of the chain-fold model. Attempts to find a consistent pattern of behavior by directly comparing $\sigma_{e}$ values amongst polymers of widely varying crosssectional areas simply obscures the basic point that $q$ is the more fundamental parameter for the purposes of comparison.

TABLE 8. Chain fold parameters for various polymers

\begin{tabular}{|c|c|c|c|}
\hline & $\begin{array}{l}\text { Polybutene-1 } \\
\text { (form 2) }\end{array}$ & Polyethylene [13] & $\begin{array}{c}\text { Polychlorotri- } \\
\text { fluoroethylene [13] }\end{array}$ \\
\hline $\begin{array}{l}\Delta h_{f}\left(\mathrm{erg} / \mathrm{cm}^{3}\right) \\
b_{0}(\mathrm{~cm}) \\
A_{0}\left(\mathrm{~cm}^{2}\right) \\
\sigma \sigma_{e}\left(\mathrm{erg}^{2} / \mathrm{cm}^{4}\right) \\
\sigma\left(\mathrm{erg} / \mathrm{cm}^{2}\right) \\
\sigma_{e}\left(\mathrm{erg} / \mathrm{cm}^{2}\right) \\
q(\mathrm{erg} / \mathrm{fold}) \\
T_{m}^{0}\left({ }^{\circ} \mathrm{K}\right)\end{array}$ & $\begin{array}{l}9.6 \times 10^{8} \\
7.45 \times 10^{-8} \\
55.4 \times 10^{-16} \\
110 \\
7.2 \\
15.5 \\
1.7 \times 10^{-13} \\
401\end{array}$ & $\begin{array}{l}2.8 \times 10^{9} \\
4.11 \times 10^{-8} \\
18.3 \times 10^{-16} \\
640 \\
11.2 \\
57 \\
2.1 \times 10^{-13} \\
415\end{array}$ & $\begin{array}{l}9.1 \times 10^{8} \\
5.6 \times 10^{-8} \\
36.4 \times 10^{-16} \\
156 \\
3.9 \\
40 \\
2.9 \times 10^{-13} \\
494\end{array}$ \\
\hline
\end{tabular}

Note in table 8 that the value of $q$ tends to be larger the higher the melting point. This is consistent with the well-known concept that an increase in chain stiffness raises $T_{m}^{0}$. A chain fold can be formed by raising about 5 bonds into gauche states [13]. The data in table 8 suggest that the equilibrium melting temperature increases with the gauche energy $\epsilon_{q} \approx q / 5$ according to $T_{m}^{0}=\Delta H_{f} / \Delta S_{f} \cong[(q / 5+C) / k \ln \Omega]$ where $C$ is a constant dependent on van der Waals forces and $\Omega=3$. A more detailed relationship between $\epsilon_{g}$ and $T_{m}^{0}$ can be devised which involves appropriate weighting factors, but the oversimplified relationship noted above is sufficient to illustrate qualitatively why increasing $q$ or $\epsilon_{g}$ tends to increase $T_{m}^{0}$.

It is significant that the above analysis in terms of the chain-folding mechanism is successful for a polymer that has a helical conformation in the crystalline state. It is also of interest to compare the above analysis with some that we have attempted (notably on certain "isotactic" specimens of poly- 
propylene and polystyrene) where there were independent reasons for suspecting that there were some "wrong," i.e., atactic, sections in the polymer chain. In these cases plots such as those shown in figure 5 were nonlinear, and absurdly low average values of $\sigma \sigma_{e}$ and $q$ were found. Some of this is probably due to underestimation of $T_{m}^{\prime}$, which is exceptionally difficult to ascertain with certainty in cases where "copolymer" type melting occurs. Similar nonlinearity and low $\sigma \sigma_{e}$ and $q$ values are obtained when an analysis is carried out on any polymer using a low $T_{m}^{0}$ value. Also, eq (6) can hardly be expected to hold precisely in the partially atactic case because sequence selection problems were left out of consideration in its derivation. Evidently, a sufficient number of the chains in the polybutene-1 used here have long stereoregular sections that crystallize in a manner analogous to that of a flexible homopolymer such as polyethylene where questions of stereoirregularity do not enter. This behavior is consistent with the high degree of crystallinity attained by the specimen of polybutene-1 (see sec. 3.2).

The value of 3 calculated for the Avrami parameter $n$ for the subcooled liquid $\rightarrow$ form 2 process in the 90 to $110{ }^{\circ} \mathrm{C}$ temperature range agrees with those calculated for polyethylene [20] and polypropylene [24]. This value is usually interpreted as indicating that either heterogeneous initiation followed by three-dimensional growth, or sporadic initiation followed by two-dimensional growth, transforms a polymer melt phase into a crystalline phase. The presence of heterogeneous nucleation is supported by photomicrographs (figs. 3 and 4) that show spherulites originating over a narrow time range at a given crystallization temperature. We therefore conclude that polybutene-1 transforms from the melt to the form 2 phase by heterogeneous nucleation followed by threedimensional growth and the analysis of the dilatometric isotherms was carried out on this assumption. Homogeneous nucleation is in any event extremely improbable except at much larger supercoolings than those attained here.

\subsection{Relative Thermodynamic Stability of Form 1 and Form 2}

Between $0{ }^{\circ} \mathrm{C}$ and its melting point, form 1 is more stable than the lower melting form 2 . Form 2 is metastable with respect to form 1 above about $60{ }^{\circ} \mathrm{C}$. A sample crystallized as form 2 at $85{ }^{\circ} \mathrm{C}$ in a dilatometer showed no change in mercury level over the period of one year. Nearer to room temperature, form 2 converts to form 1 at an observable rate, and in this region form 2 may be classed as unstable with respect to form 1 . On warming from room temperature, form 1 shows no tendency to approach the specific volume associated with form 2 either below or above the melting point of form 2 . It is clear that the shift from metastability to instability for form 2 as the temperature falls is simply a matter of increasing rate of the form $2 \rightarrow$ form 1 transformation as the temperature is lowered. In polybutene- 1 , above $0{ }^{\circ} \mathrm{C}$, there is no thermodynamic first-order phase transition between form 1 and form 2 of the type exhibited by polytetrafluorethylene near 20 and $30{ }^{\circ} \mathrm{C}$ (see sec. 1).

\subsection{Form $2 \rightarrow$ Form 1 Crystal-Crystal Transformation}

As was mentioned earlier, form 2 has an $11_{3}$ helical conformation, and form 1 has a $3_{1}$ helical conformation. The conversion from the $11_{3}$ conformation to the $3_{1}$ conformation during the form $2 \rightarrow$ form 1 transformation clearly involves an increase of density. Such an increase of density is not surprising, and forms the basis upon which we were able to follow the course of the change. The present work confirms previous observations that the form $2 \rightarrow$ form 1 transformation is not accompanied by any noticeable change in the morphological features of the spherulites. From the above information, it is reasonable to postulate that the helix change involved in the form $2 \rightarrow$ form 1 transformation propagates through a given lamella and thereby does not demand any gross reorganization of one lamella with respect to another. The nucleation term impeding the change in helicity is quite large, completely forbidding it at high temperatures, and permitting only a relatively slow rate near room temperature.

The rate of the $2 \rightarrow 1$ process can be described in terms of the reciprocal of the half-time for the transformation. This is shown in figure 6 , where similar data on the supercooled liquid $\rightarrow$ form 2 process are shown for purposes of comparison.

The subcooled liquid $\rightarrow$ form 2 transformation has already been analyzed in terms of growth governed by a coherent two-dimensional surface nucleus. The strongly negative temperature coefficient seen for the subcooled liquid $\rightarrow$ form 2 process in figure 6 is a result of the nucleation term $\exp \left[-K_{g} / T^{2}(\Delta T)\right]$ in eq (6). The interfacial transport term exp $\left[-\Delta H^{*} / R T\right]$ would at some lower temperature eventually supervene and cause the rate to fall. Thus a maximum appears in $G$ or $1 / t_{1 / 2}$ for a nucleationcontrolled process when the transport term is considered also (see for example [23]). The maximum was not seen for the supercooled liquid $\rightarrow$ form 2 process, because the rates became too rapid to measure as the temperature was lowered below about $90^{\circ} \mathrm{C}$.

The form $2 \rightarrow$ form 1 transformation exhibits the negative temperature coefficient and the maximum typical of competing nucleation-controlled and interfacial transport processes. If the form $2 \rightarrow$ form 1 transformation is in fact nucleation controlled, its much lower overall rate compared to the supercooled liquid $\rightarrow$ form 2 transformation could be attributed in part to a larger " $K_{g}$ " value. It is clear, however, that an analysis of the form $2 \rightarrow$ form 1 transformation cannot be blindly patterned after the subcooled liquid $\rightarrow$ form 2 calculation. Much more information would be needed before a detailed theory of the form $2 \rightarrow$ form 1 transformation could be advanced. 


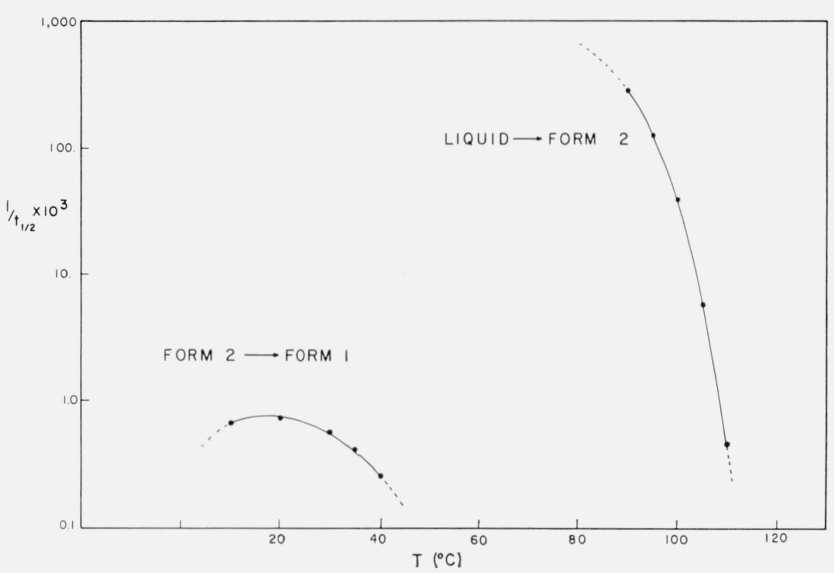

Figure 6. Reciprocallof crystallization rate half-times versus temperature for polybutene-1.

Boor and Mitchell [14] find a pronounced maximum in the rate of conversion of form 2 to form 1 between 15 and $20^{\circ} \mathrm{C}$ which is in good agreement with the present results.

The value of the Avrami growth parameter for the form $2 \rightarrow$ form 1 transformation is close to 2 . This might be interpreted as being a result of either heterogeneous initiation followed by two-dimensional growth, or sporadic initiation followed by onedimensional growth. Unlike the case of the supercooled liquid $\rightarrow$ form 2 transformation, where evidence of heterogeneous initiation was clearly apparent, the type of initiation cannot be positively identified. Boor and Mitchell [25] have observed that the addition of polypropylene or stearic acid accelerates the form $2 \rightarrow$ form 1 transformation. This indicates that the process is subject to heterogeneous initiation. While heterogeneous initiation with two-dimensional growth seems the most likely explanation of the observed value of $n$, sporadic initiation followed by one-dimensional growth cannot be completely ruled out in the case of the uncontaminated polymer.

When observed by eye alone, a freshly prepared sample of polybutene-1 (form 2) gradually becomes more turbid over the period of one week. However, it has already been noted that optical microscopy does not reveal any concomitant change in morphology or general appearance in the spherulites. Both observations might be explained by postulating that the decrease in volume caused by the form $2 \rightarrow$ form 1 transformation results in the formation of microvoids in the size range of 3000 to $6000 \AA$. Such microvoids would be too small to be resolved by an optical microscope, but would interact with visible light so as to render a polybutene-1 film turbid on visual inspection. The existence of such microvoids would naturally alter the crystallinity scale somewhat, but not to the extent of vitiating the conclusions we have based on such determinations.

The authors thank William Meyer for carrying out the viscosity measurements, and Mrs. Rosalind Newman for assisting with the spherulite studies. The financial support from the Advanced Research Projects Agency Program at the National Bureau of Standards on Crystal Growth and Defect Characterization is gratefully acknowledged.

\section{References}

[1] H. A. Rigby and C. W. Bunn, Nature 164, 583 (1949).

[2] F. A. Quinn, Jr., D. E. Roberts, and R. N. Work, J. Appl. Phys., 22, 1085 (1951).

[3] R. K. Kirby, J. Res. NBS 5\%, 91 (1956).

[4] G. Natta, Makromol. Chemie 35, 94 (1960).

[5] R. Zannetti, P. Manaresi, and G. C. Buzzoni, Chim. Ind. (Milan) 43, 735 (1961).

[6] F. A. Quinn, Jr., and J. Powers, manuscript in preparation.

[7] A Turner-Jones, J. Polymer Sci. Part B, 1, 455 (1963). See also R. L. Miller and L. E. Neilson, Appendix I in P. H. Geil, Polymer Single Crystals, Interscience, New York (1963).

[8] G. Natta, P. Corradini, and I. W. Bassi, Nuovo Cimento 15, Suppl. 1, 52 (1960).

[9] C. Geacintov, R. S. Schotland, and R. B. Miles, J. Polymer Sci. Part B, 1, 587 (1963); Part C, 6, 197 (1964).

[10] F. Danusso and G. Gianotti, Makromol. Chem. 61, 139 (1963).

[11] W. R. Krigbaum, J. E. Kurz, and P. Smith, J. Phys. Chem. 65, 1984 (1961).

[12] N. Bekkedahl, J. Res. NBS 43, 145 (1949).

[13] J. D. Hoffman, SPE Transactions 4, 315 (1964).

[14] J. Boor, Jr., and J. C. Mitchell, J. Polymer Sci. Part A, 1, 59 (1963).

[15] J. D. Hoffman and J. J. Weeks, J. Res NBS 60, 465 (1958).

[16] H. Wilski and T. Grewer, J. Polymer Sci. C6, 33 (1964).

[17] G. Natta, P. Corradini, and I. W. Bassi, Makromol. Chem. 21, 240 (1956.)

[18] G. Natta, L. Porri, P. Corradini, and D. Morero, Accad. Naz. Lincei, Rend., Classe Sci. Fis., Atti. 20, 560 (1956).

[19] R. L. Miller in Crystalline Olefin Polymers, edited by R. Raff and K. W. Doak (Interscience, New York, 1963).

[20] W. Banks, M. Gordon, R. F. Roe, and A. Sharples, Polymer 4, 61 (1963).

[21] F. J. Padden, Jr., and H. D. Keith, J. Appl. Phys. 30, $1479(1959)$.

[22] J. D. Hoffman and J. I. Lauritzen, Jr., J. Res. NBS $65 \mathbf{A}$ (Phys. and Chem.) No. 4, 297 (1961).

[23] J. D. Hoffman and J. J. Weeks, J. Chem. Phys. 37, 1723 (1962).

[24] L. Marker, P. M. Hay, G. P. Tilley, R. M. Early, and P. J. Sweeting, J. Polymer Sci. 38, 33 (1959).

[25] J. Boor, Jr., and J. C. Mitchell, J. Polymer Sci. 62, S70 (1962).

[26] V. F. Holland and R. L. Miller, J. Appl. Phys. 35, 3241 (1964).

(Paper 69A4-353) 